\title{
Aortic Pulse Wave Velocity, Ankle-Brachial Index, and Malondialdehyde in Older Adults with or without Metabolic Syndrome
}

\author{
Ruchada Sri-amad $^{a}$ Nawiya Huipao ${ }^{a}$ Piyapong Prasertsri ${ }^{\text {b }, ~ c ~}$ \\ Thapanee Roengrit ${ }^{a}$ \\ a Department of Physiology, Faculty of Science, Prince of Songkla University, Songkhla, \\ Thailand; ' ${ }^{b}$ Faculty of Allied Health Sciences, Burapha University, Chonburi, Thailand; \\ 'Exercise and Nutrition Sciences and Innovation Research Unit, Burapha University, \\ Chonburi, Thailand
}

\section{Keywords}

Metabolic syndrome $\cdot$ Arterial stiffness $\cdot$ Pulse wave velocity · Ankle-brachial index ·

Malondialdehyde

\begin{abstract}
Metabolic syndrome is an important health problem associated with both subclinical atherosclerosis and an increased risk of cardiovascular disease and it leads to an elevated total mortality. Aortic pulse wave velocity (aPWV) is widely used for noninvasive assessment of arterial stiffness. Ankle-brachial index ( $A B I$ ) predicts peripheral arterial disease (PAD) of the lower extremities. In addition, malondialdehyde (MDA) is thought to be involved in the development of arterial stiffness. The present study aimed to: (1) compare aPWV, ABI, and MDA between participants with MetS and those without MetS and (2) investigate the correlation of aPWV and $A B I$ with the components of MetS and MDA. A total of 48 Thai elderly subjects were divided into 2 groups (MetS and non-MetS) according to the parameters set by the International Diabetes Federation (IDF). aPWV and ABI were measured using the VaSera VS-1500 system (Fukuda Denshi Co., Tokyo, Japan). MDA was determined by spectrophotometry. aPWV and MDA were significantly higher in the MetS group compared to the participants in the non-MetS group $(9.33 \pm 2.72$ vs. $7.95 \pm 1.37 \mathrm{~m} / \mathrm{s}, p=0.03$, and $0.74 \pm 0.71$ vs. $0.45 \pm 0.20 \mu \mathrm{mol}$, $p=0.02$, respectively). However, $\mathrm{ABI}$ did not differ between the groups. Analysis of the risk factors of aPWV in each group revealed that there were no statistical associations between the components of MetS and MDA and aPWV in both the MetS and the non-MetS groups. A high aPWV is more prevalent among patients with MetS than among those without MetS. Monitoring of aPWV might help to explore potential detection of vascular damage in the elderly.


Sri-amad et al.: aPWV, ABI, and MDA in Older Adults with or without Metabolic Syndrome

\section{Introduction}

Metabolic syndrome (MetS) is a worldwide health problem associated with a state of chronic low-grade inflammation as a consequence of the complex interplay between genetic and environmental factors [1]. Central abdominal obesity, an elevated blood pressure (BP), hyperglycemia, and dyslipidemia are the factors that constitute the syndrome [1]. MetS appears to be linked to an increased risk of diabetes, heart disease, stroke, and other cardiovascular conditions. In addition, cardiovascular morbidity and mortality have been found to be markedly higher in patients with MetS than in those without MetS [2, 3].

Arterial stiffness caused by changes including functional alterations of vascular smooth muscle tone and structural changes in the arterial wall mainly reflects gradual fragmentation and loss of elastin fibers and accumulation of stiffer collagen fibers in the large arteries [4, 5]. Several noninvasive assessments of arterial stiffness, as measured by aortic pulse wave velocity (aPWV) and ankle-brachial index (ABI), are independently associated with all-cause and cardiovascular mortality [6]. They have been more widely used due to their accessibility, reliability, and low cost in the clinical setting. aPWV is the velocity of the BP wave as it travels a given distance between the carotid and femoral arteries [7], while the ABI is calculated as the ratio of ankle systolic BP (SBP) to brachial SBP on each side [6]. Other aspects of arterial stiffness that have been linked to MetS include endothelial dysfunction, inflammation, and oxidative stress [8]. Evidence suggests that the components of MetS (fat accumulation, dyslipidemia, and insulin resistance) are associated with an increase in the production of oxidative stress and consequently an increase in lipid peroxidation [9]. It is known that malondialdehyde (MDA) is a biomarker for oxidative stress formed during lipid peroxidation.

Numerous studies have indicated that MetS promotes arterial stiffness, which leads to the development of cardiovascular disease in humans $[3,10,11]$. However, few studies have investigated arterial stiffness on the components of MetS in the elderly who have never been treated for MetS. Therefore, the present study aimed to: (1) compare aPWV, $\mathrm{ABI}$, and MDA between MetS and non-MetS groups and (2) examine the relationship between aPWV and ABI with the components of MetS and MDA. We hypothesized that increased arterial stiffness and lipid peroxidation would occur in the MetS group. In addition, arterial stiffness might be more closely correlated with components of MetS and lipid peroxidation.

\section{Materials and Methods}

\section{Participants}

A total of 48 participants who visited the Hatyai Chivasuk Health Promotion Center from March to July 2018 were enrolled into this study. The inclusion criteria were: (1) older adults aged $\geq 60$ years with or without MetS; (2) no cardiovascular diseases such as acute myocardial infarction, stroke, and unstable angina; and (3) no intake of medication such as antihypertensive drugs, lipid-lowering drugs, or blood-sugar-lowering drugs. The exclusion criteria were: BMI $>40$ and an abnormal electrocardiogram (ECG).

\section{Diagnosis and Grouping}

After screening, the participants were divided into 2 groups (MetS $[n=24]$ and non-Mets $[n=24]$ ) based on the parameters of the International Diabetes Federation (IDF). MetS was defined as the presence of central obesity (waist circumference [WC] $\geq 90 \mathrm{~cm}$ in males and $\geq 80 \mathrm{~cm}$ in females) plus any 2 of the following criteria: (1) triglycerides (TG) $\geq 150 \mathrm{mg} / \mathrm{dL}$, (2) HDL-L $<40 \mathrm{mg} / \mathrm{dL}$ in males or $<50 \mathrm{mg} / \mathrm{dL}$ in females, (3) SBP $\geq 130 \mathrm{~mm} \mathrm{Hg}$ or diastolic BP (DBP) $\geq 85 \mathrm{~mm} \mathrm{Hg}$, and (4) fasting blood sugar (FBS) $\geq 100$ $\mathrm{mg} / \mathrm{dL}[10]$.

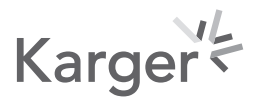




\begin{tabular}{l|l}
\hline Pulse 2020;8:31-39 \\
\hline DOI: 10.1159/000505838 & $\begin{array}{l}\text { @ 2020 S. Karger AG, Basel } \\
\text { www.karger.com/pls }\end{array}$ \\
\hline
\end{tabular}

Sri-amad et al.: aPWV, ABI, and MDA in Older Adults with or without Metabolic Syndrome

Screening Examinations

Anthropometric Measurements

Anthropometric data were collected by trained examiners in the morning. Body weight and height were measured with the participants wearing a light dress without shoes. BMI was calculated as weight (in $\mathrm{kg}$ ) divided by the square of height (in $\mathrm{m}$ ). WC was measured at the narrowest level between the lowest rib and the iliac crest, and hip circumference (HC) was measured around the widest portion of the buttocks with the use of a plastic measuring tape in the standing position; the waist-to-hip ratio was then calculated. The fat mass and the percentage of body fat were derived from the measurement of body density assessed by bioelectrical impedance analysis (UM-076 Tanita, Japan).

BP Measurement

BP was taken with the participant in a seated position with the feet flat on the floor, using an automated sphygmomanometer (Omron Health Care, Kyoto, Japan) and involved the following 4 measurements: SBP, DBP, pulse pressure (PP) (estimated as PP = SBP - DBP), and mean arterial pressure (MAP) (calculated as $\mathrm{MAP}=\mathrm{DBP}+1 / 3[\mathrm{PP}])$.

Biochemical Measurement

Blood samples ( $8 \mathrm{~mL}$ ) were collected from the antecubital vein in the morning after 10-14 h of fasting. Serum was separated immediately, and the lipid profile of the extracted serum was investigated (total cholesterol [TC], TG, high-density lipoprotein cholesterol [HDL-L], and low-density lipoprotein [LDL-C]). These were performed using the colorimetric enzymatic method. In addition, FBS was assessed using a glucosemeter (Accu-Chek ${ }^{\circledR}$ Active, Roche Diagnostics GmbH, Mannheim, Germany). Regarding the MDA assay, a blood sample ( $2 \mathrm{~mL}$ ) was taken into an EDTA tube for detection of the plasma MDA level. A marker of lipid peroxidation was assessed using the thiobarbituric acid (TBA) test according to the modified method from Ohkawa et al. [12]. The basis of the TBA method is the reaction of MDA with $0.8 \%$ of TBA at a low $\mathrm{pH}$ and $95^{\circ} \mathrm{C}$ (boiled for $60 \mathrm{~min}$ ) to form a colored complex. The MDA-TBA complex, with absorption at $532 \mathrm{~nm}$, was measured using a spectrophotometer (Genesys 10s UV-Vis; Thermo Scientific, Madison, WI, USA).

Arterial Stiffness Measurement

The aPWV and the ABI were measured in all of the participants. All measurements were automatically calculated using a VaSera VS- $1500{ }^{\circledR}$ device (Fukuda Denshi Co., Tokyo, Japan). The participants were asked to lie down and rest for $10 \mathrm{~min}$ in a quiet room at a stable temperature. Oscillometric pressure cuffs were placed approximately $2 \mathrm{~cm}$ above the antecubital fossa on the arms and approximately $2 \mathrm{~cm}$ above the medial malleolus on the ankles. ECG electrodes were attached to both wrists and a pulse wave sensor was placed on the carotid and the femoral arteries. A microphone for phonocardiography was placed in the second intercostal space on the left edge of the sternum to listen for the second heart sound. The wave form analyzer automatically determines the aPWV. The aPWV was obtained using the formula PWV = D/ $\Delta \mathrm{t}(\mathrm{m} / \mathrm{s})$, where $\Delta \mathrm{t}$ was determined based on the time delay of the waveform between the carotid and femoral arteries. The distance (D) traveled by the pulse was estimated based on the height, age, and gender. An aPWV $>10 \mathrm{~m} / \mathrm{s}$ has been estimated to be a significant alteration of aortic function [7]. ABI measurements were determined from both left and right arms and ankles. The ratio of the SBP in the ankle to the arm defined the ABI. Participants with an ABI $<0.9$ were suspected to have obstructive arteriosclerosis [13].

\section{Statistical Analysis}

Statistical analyses were carried out using SPSS. Categorical variables (gender, participant's ABI $<0.9$, and $\mathrm{aPWV}>10 \mathrm{~m} / \mathrm{s}$ ) were described as numbers (\%). Continuous variables (age, hemodynamic parameters, biochemical parameters, $\mathrm{ABI}$, and $\mathrm{aPWV}$ ) were expressed as means $\pm \mathrm{SD}$. The $\chi^{2}$ test was used to compare categorical variables. The Shapiro-Wilk test was applied to examine the normal distribution of the data. To compare normally distributed data (resting heart rate, SBP, DBP, PP, HC, fat mass, percentage of body fat, TC, HDL-C, LDL-C, R-ABI, and L-ABI), an independent $t$ test was utilized; otherwise the Mann-Whitney U-test was applied. Correlations between variables were assessed by Pearson's (normally distributed data) and Spearman's rank (non-normally distributed data) correlation coefficient. Multiple linear regression analysis was used to identify the risk factors of aPWV in the MetS and non-MetS groups. $\mathrm{p}<0.05$ was considered statistically significant.

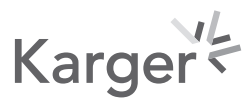




\begin{tabular}{l|l}
\hline Pulse 2020;8:31-39 \\
\hline DOI: 10.1159/000505838 & $\begin{array}{l}\text { (c) 2020 S. Karger AG, Basel } \\
\text { www.karger.com/pls }\end{array}$ \\
\hline
\end{tabular}

Sri-amad et al.: aPWV, ABI, and MDA in Older Adults with or without Metabolic Syndrome

Table 1. Baseline characteristics and hemodynamic, anthropometric, and biochemical parameters of the study population

\begin{tabular}{|c|c|c|c|}
\hline \multirow[t]{2}{*}{ Variable } & \multicolumn{3}{|l|}{ Value } \\
\hline & $\begin{array}{l}\text { non-MetS } \\
(n=24)\end{array}$ & $\begin{array}{l}\text { MetS } \\
(n=24)\end{array}$ & $p$ value \\
\hline Age, years & $67.71 \pm 4.60$ & $67.42 \pm 4.17$ & 0.82 \\
\hline Males & $3(12.5)$ & $7(29.2)$ & 0.21 \\
\hline \multicolumn{4}{|l|}{ Hemodynamic parameters } \\
\hline Resting heart rate, beats/min & $67.50 \pm 9.82$ & $69.92 \pm 8.93$ & 0.38 \\
\hline SBP, mm Hg & $131.83 \pm 17.05$ & $139.83 \pm 15.46$ & 0.10 \\
\hline DBP, mm Hg & $79.04 \pm 7.21$ & $80.92 \pm 7.97$ & 0.40 \\
\hline $\mathrm{PP}, \mathrm{mm} \mathrm{Hg}$ & $52.79 \pm 13.41$ & $58.92 \pm 13.93$ & 0.13 \\
\hline MAP, mm Hg & $89.13 \pm 6.10$ & $94.67 \pm 9.32$ & $0.02 *$ \\
\hline \multicolumn{4}{|l|}{ Anthropometric parameters } \\
\hline Height, cm & $157.25 \pm 8.92$ & $155.21 \pm 8.18$ & 0.41 \\
\hline Weight, kg & $57.06 \pm 7.15$ & $62.50 \pm 8.26$ & $0.02 *$ \\
\hline BMI & $23.17 \pm 3.38$ & $26.31 \pm 3.65$ & $0.02 *$ \\
\hline $\mathrm{WC}, \mathrm{cm}$ & $81.42 \pm 5.63$ & $92.04 \pm 8.76$ & $<0.01 *$ \\
\hline $\mathrm{HC}, \mathrm{cm}$ & $95.63 \pm 5.30$ & $99.75 \pm 5.10$ & $0.01^{*}$ \\
\hline WHR & $0.85 \pm 0.06$ & $0.92 \pm 0.07$ & $<0.01^{*}$ \\
\hline Fat mass, kg & $17.01 \pm 3.66$ & $21.00 \pm 4.58$ & $<0.01^{*}$ \\
\hline Lean body mass, kg & $40.05 \pm 4.61$ & $41.50 \pm 6.17$ & 0.36 \\
\hline Body fat, $\%$ & $29.60 \pm 3.96$ & $33.50 \pm 5.17$ & $0.01 *$ \\
\hline \multicolumn{4}{|l|}{ Biochemical parameters } \\
\hline $\mathrm{TC}, \mathrm{mg} / \mathrm{dL}$ & $222.50 \pm 52.00$ & $234.92 \pm 44.84$ & 0.38 \\
\hline HDL-C, mg/dL & $71.25 \pm 17.33$ & $57.96 \pm 18.76$ & $0.01 *$ \\
\hline $\mathrm{TG}, \mathrm{mg} / \mathrm{dL}$ & $81.83 \pm 26.20$ & $131.17 \pm 58.33$ & $<0.01 *$ \\
\hline $\mathrm{LDL}-\mathrm{C}, \mathrm{mg} / \mathrm{dL}$ & $147.68 \pm 47.81$ & $159.10 \pm 40.74$ & 0.38 \\
\hline FBS, mg/dL & $95.08 \pm 6.28$ & $117.33 \pm 31.14$ & $<0.01^{*}$ \\
\hline $\mathrm{MDA}, \mu \mathrm{mol}$ & $0.45 \pm 0.20$ & $0.74 \pm 0.71$ & $0.02 *$ \\
\hline \multicolumn{4}{|l|}{ Arterial stiffness parameters } \\
\hline R-ABI & $1.11 \pm 0.07$ & $1.09 \pm 0.09$ & 0.35 \\
\hline L-ABI & $1.10 \pm 0.06$ & $1.10 \pm 0.08$ & 0.95 \\
\hline $\mathrm{ABI}<0.9$ & $3(12.5)$ & $2(8.3)$ & 0.65 \\
\hline $\mathrm{aPWV}, \mathrm{m} / \mathrm{s}$ & $7.95 \pm 1.37$ & $9.33 \pm 2.72$ & $0.03 *$ \\
\hline $\mathrm{aPWV}>10, \mathrm{~m} / \mathrm{s}$ & $2(8.3)$ & $8(33.3)$ & 0.06 \\
\hline
\end{tabular}

Categorical variables are presented as numbers (\%). Continuous variables are presented as means \pm SD. HC, hip circumference; WHR, waist-to-hip ratios. ${ }^{*} p<0.05$.

\section{Results}

Baseline Characteristics and Hemodynamic and Anthropometric Parameters

Of the 48 participants, $20.83 \%$ were male and $79.17 \%$ were female. They were divided into an MetS group ( $n=24, \mathrm{M}: \mathrm{F}$ ratio $=7: 17$, mean age $=67.42 \pm 4.17$ years $)$ and a non-MetS group $(n=24, \mathrm{M}: \mathrm{F}$ ratio $=3: 21$, mean age $=67.71 \pm 4.60$ years). Age did not differ between the groups. However, the MAP was significantly higher in the MetS group than in the non-MetS group ( $94.67 \pm 9.32$ vs. $89.13 \pm 6.10 \mathrm{~mm} \mathrm{Hg}, p=0.02$ ). Anthropometric parameters (except for height and lean body mass) were significantly higher in the MetS group than in the non-MetS group (Table 1). 
Table 2. Correlation between aPWV with the components of MetS and MDA of the study population

\begin{tabular}{|c|c|c|c|c|c|c|}
\hline \multirow[t]{2}{*}{ Variables } & \multicolumn{2}{|c|}{$\begin{array}{l}\text { All participants } \\
(n=48)\end{array}$} & \multicolumn{2}{|c|}{$\begin{array}{l}\text { Non-MetS } \\
(n=24)\end{array}$} & \multicolumn{2}{|c|}{$\begin{array}{l}\text { MetS } \\
(n=24)\end{array}$} \\
\hline & $r$ & $p$ value & $r$ & $p$ value & $r$ & $p$ value \\
\hline SBP, mm Hg & 0.30 & $0.03^{*}$ & 0.03 & 0.87 & 0.40 & 0.05 \\
\hline DBP, mm Hg & 0.15 & 0.28 & 0.21 & 0.30 & 0.09 & 0.67 \\
\hline WC, $\mathrm{cm}$ & 0.39 & $0.01^{*}$ & 0.37 & 0.06 & 0.30 & 0.14 \\
\hline HDL-C, mg/dL & -0.17 & 0.24 & 0.15 & 0.48 & -0.07 & 0.72 \\
\hline TG, mg/dL & 0.01 & 0.93 & -0.21 & 0.31 & -0.15 & 0.46 \\
\hline $\mathrm{FBS}, \mathrm{mg} / \mathrm{dL}$ & 0.39 & $0.01^{*}$ & 0.12 & 0.55 & 0.46 & $0.02^{*}$ \\
\hline MDA, $\mu \mathrm{mol}$ & 0.28 & 0.05 & -0.26 & 0.20 & 0.45 & $0.02 *$ \\
\hline
\end{tabular}

${ }^{*} p<0.05$

Fig. 1. aPWV of the study population. Data are presented as means \pm SD; $n=24$ for each group. NonMetS and MetS groups are indicated by open and closed bars, respectively.

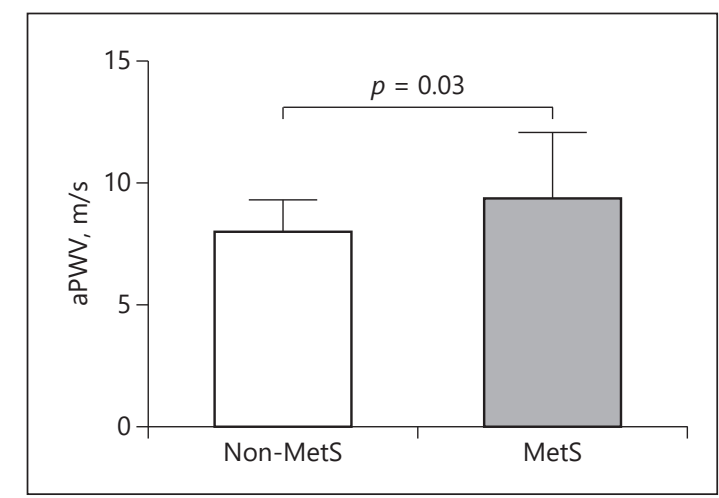

\section{Biochemical Parameters}

HDL-C was significantly lower in the MetS group than in the non-MetS group (57.96 \pm 18.76 vs. $71.25 \pm 17.33 \mathrm{mg} / \mathrm{dL}, p=0.01$ ). However, TG, FBS, and MDA were significantly higher in the MetS group than in the non-MetS group $(131.17 \pm 58.33 \mathrm{vs} .81 .83 \pm 26.20 \mathrm{mg} /$ $\mathrm{dL}, p<0.01 ; 117.33 \pm 31.14$ vs. $95.08 \pm 6.28 \mathrm{mg} / \mathrm{dL}, p<0.01$; and $0.74 \pm 0.71$ vs. $0.45 \pm 0.20$ $\mu \mathrm{mol}, p=0.02$, respectively). There was no significant difference between groups in terms of TC and LDL-C (Table 1).

\section{Arterial Stiffness Parameters}

aPWV was significantly higher in the MetS group compared to the non-MetS group (9.33 \pm 2.72 vs. $7.95 \pm 1.37 \mathrm{~m} / \mathrm{s}, p=0.03$ ) (Table 1 ; Fig. 1), while ABI did not differ between the groups (Table 1).

Association between Arterial Stiffness Parameters and the Components of MetS and MDA

In the MetS group, aPWV was positively correlated with FBS and MDA ( $r=0.46, p=0.02$; $r=0.45, p=0.02$ ) (Table 2). However, there was no correlation between aPWV and BP, WC, and lipid profile. ABI was not correlated with the components of MetS and MDA (data not shown).

The analysis of the risk factors of aPWV in each group is shown in Table 3. There were no statistical associations between the components of MetS and MDA and aPWV in the MetS and non-MetS groups. 


\begin{tabular}{l|l}
\hline Pulse 2020;8:31-39 \\
\hline DOI: 10.1159/000505838 & $\begin{array}{l}\text { @ 2020 S. Karger AG, Basel } \\
\text { www.karger.com/pls }\end{array}$ \\
\hline
\end{tabular}

Sri-amad et al.: aPWV, ABI, and MDA in Older Adults with or without Metabolic Syndrome

Table 3. Multiple linear regression analysis for aPWV

\begin{tabular}{|c|c|c|c|c|c|c|}
\hline \multirow[t]{2}{*}{ Variables } & \multicolumn{2}{|c|}{$\begin{array}{l}\text { All participants } \\
(n=48)\end{array}$} & \multicolumn{2}{|c|}{$\begin{array}{l}\text { Non-MetS } \\
(n=24)\end{array}$} & \multicolumn{2}{|c|}{$\begin{array}{l}\text { MetS } \\
(n=24)\end{array}$} \\
\hline & $\beta$ & $p$ value & $\beta$ & $p$ value & $\beta$ & $p$ value \\
\hline SBP, mm Hg & -0.02 & 0.86 & -0.15 & 0.63 & 0.32 & 0.17 \\
\hline DBP, mm Hg & -0.04 & 0.76 & -0.03 & 0.92 & 0.10 & 0.64 \\
\hline $\mathrm{WC}, \mathrm{cm}$ & -0.01 & 0.93 & 0.14 & 0.54 & 0.43 & 0.05 \\
\hline HDL-C, mg/dL & 0.16 & 0.27 & -0.44 & 0.11 & -0.06 & 0.80 \\
\hline $\mathrm{TG}, \mathrm{mg} / \mathrm{dL}$ & -0.01 & 0.90 & -0.17 & 0.54 & -0.23 & 0.30 \\
\hline FBS, mg/dL & 0.02 & 0.87 & 0.03 & 0.86 & 0.07 & 0.71 \\
\hline MDA, $\mu$ mol & 0.01 & 0.94 & -0.00 & 0.99 & 0.24 & 0.24 \\
\hline
\end{tabular}

Multiple linear regression analyses were performed to determine the components of MetS and MDA associated with aPWV. $\beta$, unstandardized regression coefficient.

\section{Discussion/Conclusion}

MetS consists of visceral obesity, dyslipidemia, hypercholesterinemia, and elevated BP and fasting plasma glucose. Several studies have also reported that MetS and all its components are associated with increased arterial stiffness [5, 11, 14], leading to increased aPWV. The possible mechanisms of high aPWV among people with MetS include the following: (1) vascular structural changes as a result of chronic hyperglycemia. The present study found that the FBS value showed a statistically significant difference between the 2 groups and a positive correlation with aPWV among the patients with MetS. It is known that hyperglycemia is linked to an increase in the local activity of the renin-angiotensin-aldosterone system and expression of angiotensin II (AT II). AT II plays an important role through the AT1 receptor, induces proliferation of vascular smooth muscle cells (VSMC), and increases collagen synthesis [1]. These changes in the vascular wall may lead to the development of arterial stiffness. (2) Impaired glucose tolerance enhances nonenzymatic advanced glycation end products on the vascular matrix proteins, with a consequent increase in collagen fiber production in the vascular wall [15], leading to generation of oxidative stress or interaction with membrane receptors triggering the inflammatory process [16]. A chronic inflammatory process in MetS and all of its components, which activates extracellular matrix metalloproteinases, is thought to contribute to alterations in vascular collagen and/or elastin content in the arterial wall, proliferation and migration of VSMC, and plaque rupture, leading to vascular remodeling and arterial stiffness [17].

The present study showed that aPWV indicating arterial stiffness in elderly persons with MetS was higher than in elderly persons without MetS. Similar findings were also obtained in the previous study, in which aPWV was significantly higher in the MetS group compared to the non-MetS group [7]. Interestingly, aPWV has been used for the gold standard assessment of central arterial stiffness [18]. It is well established that the aPWV corresponds to systemic vascular aging, vascular elasticity, and PP amplification, which are significantly and independently associated with vascular damage [19]. aPWV $>10 \mathrm{~m} / \mathrm{s}$ has been suggested as a conservative estimate of significant alterations of aortic function [7]. Thus, aPWV measurement would be an important strategy for the identification of people at a high cardiovascular risk. Elevated aPWV is also associated with pathological factors such as old age, hypertension, and arteriosclerosis, which are directly linked to cardiovascular morbidity and mortality [20,21]. Our study procedure and results are similar to those of Kim et al. [21], who found the baPWV 
Sri-amad et al.: aPWV, ABI, and MDA in Older Adults with or without Metabolic Syndrome

values were higher in MetS subjects than in non-Mets subjects and that baPWV was positively related with age. However, we further investigated the differences among elderly individuals with and without MetS. Moreover, ABI was also analyzed to determine peripheral vascular degeneration and the cardiovascular risk independently of traditional cardiovascular risk factors.

High SBP and DBP are the components of MetS. However, the present study found no difference in SBP and DBP between the groups. Moreover, BP was not correlated with aPWV. Inconsistent with previous studies, these studies revealed that the effect of a high BP on progressive arterial stiffness is mediated by its direct effect on endothelial cells and arterial smooth muscle cells in patients with MetS $[2,11,22]$. There is a greater recruitment of collagen fibers and a reduction in vascular elasticity [23], which reflects an increased aPWV. Moreover, aPWV was correlated significantly with SBP, MAP, and PP but not with DBP in a study of patients with peritoneal dialysis [24]. Other studies have found a strong relation between DBP and aPWV in young healthy males [25]. PP has the strongest correlation with aPWV in normotensive and hypertensive subjects [26]. The controversial results on the relation between aPWV and each of the BP parameters were explained by the different demographic characteristics of the study populations such as age, gender, and body size. Moreover, the different methods and locations of measurement may also affect the results [26].

Oxidative stress and chronic low-grade inflammation are common comorbidities of MetS. Our study showed that plasma MDA was significantly higher in the MetS group compared to the non-MetS group. In our study, MDA was not significantly associated with aPWV in patients with MetS. This is inconsistent with a previous study, which indicated a high MDA level in an elderly woman with MetS [9]. MDA is known to generate secondary products of lipid peroxidation, reflecting the magnitude of the oxidative stress present in the organism [27]. Furthermore, the MetS prevalence was greater in participants with higher plasma MDA concentrations [28]. The mechanism that leads to a higher aPWV with increased MDA is still unclear. There are possible mechanisms linking oxidative stress and arterial stiffness. Enhanced generation of reactive oxygen species within the vascular wall may be responsible for vascular remodeling, contributing to the proliferation of VSMC, which leads to endothelial dysfunction $[29,30]$. Thus, all of the mechanisms that reduce vascular distensibility lead to a faster aPWV.

In this study we also determined ABI, which is the index for predicting subclinical PAD [31]. The previous study found a relationship between a low ABI and high all-cause and cardiovascular disease mortality in patients with MetS [32]. However, the unchanged ABI in this study may be explained by the fact that ABI is likely to be unreliable because of falsely increased values, or a marked decrease in ankle pressure measurement, caused by medial arterial calcification and incompressibility in advanced-age subject [33]. Meanwhile, because of interference with circulation through the lower extremities, aPWV cannot be estimated properly in patients with PAD $[13,34]$. Thus, when estimating the significance of arterial stiffness, it is necessary to exclude patients with PAD.

The present study had some limitations. Firstly, we measured parameters in only a small number of participants. Furthermore, differences in the study population such as gender, genetic factors, health status, lifestyle characteristics, and others could impact arterial stiffness. Thus, a large population is required with adjustment for possible confounding. Secondly, we did not directly measure the vascular path length. The arterial distance between recording sites was estimated using only calculations from height, age, and gender.

A high aPWV is more prevalent among patients with MetS than among those without MetS. Hence, aPWV has potential as a noninvasive tool for the detection of vascular stiffness. Monitoring of aPWV might be more useful for early detection of vascular damage in certain

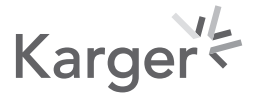


Sri-amad et al.: aPWV, ABI, and MDA in Older Adults with or without Metabolic Syndrome

populations, such as those with cardiovascular risk or other vascular abnormalities. However, it seems that the clinical interpretation and availability of these techniques is largely limited, and additional research is needed to clarify and explain the aPWV value.

\section{Acknowledgement}

We thank all of the participants for their participation in this study, all of the staff at the Hatyai Chivasuk Health Promotion Center for their technical assistance, and the Publication Clinic and Dr. Alan Geater for English proofreading of the manuscript.

\section{Statement of Ethics}

All of the participants provided written informed consent according to the general recommendations of the Declaration of Helsinki prior to performance of any of the study procedures. This study was approved by the Research Ethics Committees of the Faculty of Medicine of the Prince of Songkla University (60-166-19-2).

\section{Disclosure Statement}

The authors have no conflict of interests to declare.

\section{Funding Sources}

This work was supported by grants from the Research and Development Office (RDO; contract No. SCI610431S), Prince of Songkla University.

\section{Author Contributions}

R.S., N.H., and T.R. carried out the experiment. T.R. and P.P. contributed to the interpretation of the results. N.H., T.R., and P.P. wrote the manuscript. T.R. conceived the original idea and supervised the project.

\section{References}

1 Kaur J. A comprehensive review on metabolic syndrome. Cardiol Res Pract. 2014;2014:943162.

2 Li CI, Kardia SL, Liu CS, Lin WY, Lin CH, Lee YD, et al. Metabolic syndrome is associated with change in subclinical arterial stiffness: a community-based Taichung community health study. BMC Public Health. 2011 Oct;11(1):808.

3 Maruhashi T, Soga J, Fujimura N, Idei N, Mikami S, Iwamoto Y, et al. Endothelial Dysfunction, Increased Arterial Stiffness, and Cardiovascular Risk Prediction in Patients With Coronary Artery Disease: FMD-J (Flow-Mediated Dilation Japan) Study A. J Am Heart Assoc. 2018 Jul;7(14):e008588.

4 Oh YS. Arterial stiffness and hypertension. Clin Hypertens. 2018 Dec;24(1):17.

5 Topouchian J, Labat C, Gautier S, Bäck M, Achimastos A, Blacher J, et al. Effects of metabolic syndrome on arterial function in different age groups: the Advanced Approach to Arterial Stiffness study. J Hypertens. 2018 Apr;36(4):824-33.

6 Li M, Xin J, Kuang DW, Kuok UI, Li CL, Cheung K, et al. Risk factors of arterial damage assessed by ABI and baPWV among hemodialysis patients in Macau. Adv Neurol. 2014;2014:6.

7 Lee DH, Youn HJ, Chung WB, Choi YS, Lee JM, Park CS, et al. Effects of metabolic syndrome on aortic pulse wave velocity. Clin Hypertens. 2017 Jan;23(1):1.

8 Roberts CK, Hevener AL, Barnard RJ. Metabolic syndrome and insulin resistance: underlying causes and modification by exercise training. Compr Physiol. 2013 Jan;3(1):1-58.

9 Bakhtiari A, Hajian-Tilaki K, Omidvar S, Nasiri Amiri F. Association of lipid peroxidation and antioxidant status with metabolic syndrome in Iranian healthy elderly women. Biomed Rep. 2017 Oct;7(4):331-6. 


\begin{tabular}{l|l}
\hline Pulse 2020;8:31-39 \\
\hline DOI: 10.1159/000505838 & $\begin{array}{l}\text { @ 2020 S. Karger AG, Basel } \\
\text { www.karger.com/pls }\end{array}$ \\
\hline
\end{tabular}

Sri-amad et al.: aPWV, ABI, and MDA in Older Adults with or without Metabolic Syndrome

10 Weng C, Yuan H, Tang X, Huang Z, Yang K, Chen W, et al. Age- and gender dependent association between components of metabolic syndrome and subclinical arterial stiffness in a Chinese population. Int J Med Sci. 2012;9(8):730-7.

11 Scuteri A, Cunha PG, Rosei EA, Badariere J, Bekaert S, Cockcroft JR, et al.; MARE Consortium. Arterial stiffness and influences of the metabolic syndrome: a cross-countries study. Atherosclerosis. 2014 Apr;233(2):654-60.

12 Ohkawa H, Ohishi N, Yagi K. Assay for lipid peroxides in animal tissues by thiobarbituric acid reaction. Anal Biochem. 1979 Jun;95(2):351-8.

13 Kitahara T, Ono K, Tsuchida A, Kawai H, Shinohara M, Ishii Y, et al. Impact of brachial-ankle pulse wave velocity and ankle-brachial blood pressure index on mortality in hemodialysis patients. Am J Kidney Dis. 2005 Oct; 46(4):688-96.

14 Gomez-Sanchez L, Garcia-Ortiz L, Patino-Alonso MC, Recio-Rodriguez JI, Fernando R, Marti R, et al.; MARK Group. Association of metabolic syndrome and its components with arterial stiffness in Caucasian subjects of the MARK study: a cross-sectional trial. Cardiovasc Diabetol. 2016 Oct;15(1):148.

15 Safar ME, Czernichow S, Blacher J. Obesity, arterial stiffness, and cardiovascular risk. J Am Soc Nephrol. 2006 Apr;17(4 Suppl 2):S109-11.

16 Guéraud F, Atalay M, Bresgen N, Cipak A, Eckl PM, Huc L, et al. Chemistry and biochemistry of lipid peroxidation products. Free Radic Res. 2010 Oct;44(10):1098-124.

17 Sun Z. Aging, arterial stiffness, and hypertension. Hypertension. 2015 Feb;65(2):252-6.

18 Barodka VM, Joshi BL, Berkowitz DE, Hogue CW Jr, Nyhan D. Review article: implications of vascular aging. Anesth Analg. 2011 May;112(5):1048-60.

19 Miyatani M, Masani K, Oh PI, Miyachi M, Popovic MR, Craven BC. Pulse wave velocity for assessment of arterial stiffness among people with spinal cord injury: a pilot study. J Spinal Cord Med. 2009;32(1):72-8.

20 Cruickshank K, Riste L, Anderson SG, Wright JS, Dunn G, Gosling RG. Aortic pulse-wave velocity and its relationship to mortality in diabetes and glucose intolerance: an integrated index of vascular function? Circulation. 2002 Oct;106(16):2085-90.

21 Kim M, Kim M, Yoo HJ, Lee SY, Lee SH, Lee JH. Age-Specific Determinants of Pulse Wave Velocity among Metabolic Syndrome Components, Inflammatory Markers, and Oxidative Stress. J Atheroscler Thromb. 2018 Feb; 25(2):178-85.

22 Lee HY, Oh BH. Aging and arterial stiffness. Circulation. 2010 Nov;74(11):2257-62.

23 Quinn U, Tomlinson LA, Cockcroft JR. Arterial stiffness. JRSM Cardiovasc Dis. 2012 Sep;1(6):cvd.2012.012024.

24 Stompór T, Rajzer M, Sułowicz W, Dembińska-Kieć A, Janda K, Kawecka-Jaszcz K, et al. An association between aortic pulse wave velocity, blood pressure and chronic inflammation in ESRD patients on peritoneal dialysis. Int J Artif Organs. 2003 Mar;26(3):188-95.

25 Nürnberger J, Dammer S, Opazo Saez A, Philipp T, Schäfers RF. Diastolic blood pressure is an important determinant of augmentation index and pulse wave velocity in young, healthy males. J Hum Hypertens. 2003 Mar; 17(3):153-8.

26 Kim EJ, Park CG, Park JS, Suh SY, Choi CU, Kim JW, et al. Relationship between blood pressure parameters and pulse wave velocity in normotensive and hypertensive subjects: invasive study. J Hum Hypertens. 2007 Feb; 21(2):141-8.

27 Moreto F, Kano HT, Torezan GA, de Oliveira EP, Manda RM, Teixeira O, et al. Changes in malondialdehyde and C-reactive protein concentrations after lifestyle modification are related to different metabolic syndromeassociated pathophysiological processes. Diabetes Metab Syndr. 2015 Oct-Dec;9(4):218-22.

28 Moreto F, de Oliveira EP, Manda RM, Burini RC. The higher plasma malondialdehyde concentrations are determined by metabolic syndrome-related glucolipotoxicity. Oxid Med Cell Longev. 2014;2014:505368.

29 Arrebola-Moreno AL, Laclaustra M, Kaski JC. Noninvasive assessment of endothelial function in clinical practice. Rev Esp Cardiol (Engl Ed). 2012 Jan;65(1):80-90.

30 Lessiani G, Santilli F, Boccatonda A, Iodice P, Liani R, Tripaldi R, et al. Arterial stiffness and sedentary lifestyle: role of oxidative stress. Vascul Pharmacol. 2016 Apr;79:1-5.

31 Zhang Y, Chen J, Zhang K, Kong M, Wang T, Chen R, et al. Inflammation and oxidative stress are associated with the prevalence of high aankle-brachial index in metabolic syndrome patients without chronic renal failure. Int J Med Sci. 2013;10(2):183-90.

32 Xu Y, Li J, Luo Y, Wu Y, Zheng L, Yu J, et al. The association between ankle-brachial index and cardiovascular or all-cause mortality in metabolic syndrome of elderly Chinese. Hypertens Res. 2007 Jul;30(7):613-9.

33 Takahara M, Fujiwara Y, Katakami N, Sakamoto F, Kaneto H, Matsuoka TA, et al. Shared and additional risk factors for decrease of toe-brachial index compared to ankle-brachial index in Japanese patients with diabetes mellitus. Atherosclerosis. 2014 Jul;235(1):76-80.

34 Ikura K, Hanai K, Oka S, Watanabe M, Oda Y, Hamada M, et al. Brachial-ankle pulse wave velocity, but not anklebrachial index, predicts all-cause mortality in patients with diabetes after lower extremity amputation. J Diabetes Investig. 2017 Mar;8(2):250-3. 